\title{
Análisis etnográfico en salas de espera de centros de salud primaria: implicancias para la detección y tratamiento de la depresión posparto
}

\section{Ethnographic analysis at general practice waiting rooms: implications for postpartum depression detection and treatment}

\author{
Pablo Martínez \\ Hospital Clínico, Facultad de Medicina, Universidad de Chile \\ Escuela de Psicología, Facultad de Humanidades, Universidad de Santiago de Chile \\ Graciela Rojas \\ Hospital Clínico, Facultad de Medicina, Universidad de Chile \\ Irene Magaña \\ Escuela de Psicología, Facultad de Humanidades, Universidad de Santiago de Chile
}

(Rec: 25 abril 2014 / Acept: 3 julio 2014)

\begin{abstract}
Resumen
Chile cuenta con tamizaje y acceso universal para el tratamiento de la depresión posparto, no obstante, ésta es una patología sub-detectada y sub-tratada. Con el objetivo de describir y analizar las barreras de acceso para la detección y tratamiento de la depresión posparto implicadas en la producción de cuidados maternoinfantiles en las salas de espera de consultorios, se llevó a cabo un estudio etnográfico en seis centros de salud primaria Santiago de Chile. La maternidad, como objeto de discursos y prácticas reproducidos en los espacios comunes del consultorio, se constituye en una condición conflictiva para las puérperas, implicando una sobrecarga diferencial de trabajo doméstico y de cuidados, exigiendo su movilización entre instancias formales e informales del cuidado de la salud. Se problematiza en torno al grado de integración entre trabajo remunerado y el cuidado de la salud en las mujeres.

Palabras clave: depresión posparto, antropología cultural, accesibilidad a servicios de salud, estudios de la mujer y cuidados.
\end{abstract}

\begin{abstract}
In Chile, universal screening and treatment for postpartum depression has been implemented at a national level, however it remains under-detected and under-treated. To describe and analyze barriers to postpartum depression detection and treatment implied in maternal-infant health care production at general practice waiting rooms, an ethnographic study was carried out at six primary care clinics belonging to the Metropolitan Area of Santiago, Chile. Discourses and practices on maternity enacted at waiting room, reveals a conflicting condition for postpartum women, implying a differential overburden with domestic work and care tasks, demanding constant mobilization between formal and informal health care sources. The grade of integration between remunerated work and women's self-care is debated.

Keywords: postpartum depression, cultural anthropology, health services accessibility, women's studies, nursing.
\end{abstract}

\footnotetext{
Correspondencia: Pablo Martínez Díaz, Av. La Paz 1003, Recoleta, Región Metropolitana, Santiago, Chile. Correo electrónico: pablo88.martinezdiaz@gmail.com. Este estudio contó con el apoyo de la Iniciativa Científica Milenio del Ministerio de Economía, Fomento y Turismo de Chile, Proyecto NS100018.
} 


\section{Introducción}

En Chile, durante la última década, la depresión ha sido reconocida como problema de salud pública, estructurándose un programa con base en la atención primaria (APS) del sistema de salud público (Ministerio de Salud, s.f), y siendo incluida en el Régimen de Garantías Explícitas en Salud (Ministerio de Salud, 2006), que promueve la cobertura efectiva de la patología.

Las usuarias de este sistema suelen ser mujeres en edad fértil y de estrato socioeconómico bajo (Drago, 2006), determinante social que estructura la vida de mujeres en sectores populares, exponiéndolas diferencialmente a situaciones de vulnerabilidad, pudiendo resultar en una marcada propensión a padecer trastornos mentales comunes, en especial depresión (Araya, Rojas, Fritsch, Acuña y Lewis, 2001; Busfield, 1998; Patel, Araya, de Lima, Ludermir y Todd, 1999).

En este contexto, las construcciones discursivas en torno al sexo femenino y a la maternidad imponen desafíos adicionales para las madres de escasos recursos, estableciendo e institucionalizando inequidades, constituyéndolas en base social exclusiva de políticas sanitarias al asumir su disponibilidad para efectuar tareas de cuidados domésticos, dificultando, por consiguiente, su incorporación al trabajo remunerado (Magaña, Calquín, Silva y García, 2011; Matamala, Eguiguren y Díaz, 2011; Provoste y Berlagoscky, 2002).

A esto se suma la carga cotidiana de eventos adversos que pueden vivir durante el período perinatal y que adquieren especial significación (conflictos con la pareja, separación, estrechez económica, dificultades en cuidado de otros hijos etc.), viendo comprometida su capacidad de respuesta, encontrándose en un mayor riesgo de depresión en el posparto (DPP) (Robertson, Grace, Wallington y Stewart, 2004).

La DPP es una enfermedad prevalente, que afecta a una de cada cinco beneficiarias de la APS durante los primeros meses del posparto (Alvarado et al., 2000; Chile Crece Contigo-Ministerio de Salud, 2013), asociándose a grados importantes de discapacidad (Rojas, Fritsch, Solís, González, Guajardo y Araya, 2006), concentrándose en madres que sufren diversas formas de estrés psicosocial y con antecedentes mórbidos de salud mental (Alvarado et al., 2000; Chile Crece Contigo-Ministerio de Salud, 2013; Jadresic y Araya, 1995; Póo, Espejo, Godoy, Gualda, Hernández y Pérez, 2008; Risco, Jadresic, Galleguillos, Garay, González y Hasbún, 2002; Rojas et al., 2006; Rojas, Fritsch, Guajardo, Rojas, Barroilhet y Jadresic, 2010).

Ante este panorama, el Ministerio de Salud (MINSAL) ha recomendado la aplicación de un tamizaje universal para incentivar la detección temprana de posibles casos (Ministerio de Salud, 2013), no obstante, parecen existir serias limitaciones en el acceso al tratamiento de mujeres con DPP (Chile Crece Contigo-Ministerio de Salud, 2013), a pesar de que se ha probado la efectividad de una intervención de múltiples componentes (Rojas et al., 2007).

Las graves consecuencias que esta enfermedad puede traer para el vínculo madre-hijo, el desarrollo infantil y la salud mental materna (Wachs, Black y Engle, 2009), debieran ser motivos suficientes para explorar las causas que subyacen a este fenómeno. Al respecto, la literatura internacional sugiere la presencia de barreras en el sistema de salud, en los/las profesionales y en las mujeres mismas, factores que influyen negativamente en el uso de servicios (Dennis y Chung-Lee, 2006).

Es importante destacar que si bien estas categorías (sistema de salud, profesionales y mujeres) resultan útiles para describir los elementos involucrados en las bajas tasas de diagnóstico y tratamiento de la DPP, no se han articulado bajo una lógica de proceso, limitando el análisis de sus relaciones e impidiendo problematizar posibles determinantes estructurales. En este sentido, la aplicación del concepto de 'elegibilidad' (Dixon-Woods et al., 2005) puede arrojar luces sobre cómo se imbrican estas barreras de acceso a los cuidados institucionalizados de la salud, ya que considera la atención de la salud como un proceso coherente, contingente y relacional, negociado entre las usuarias y los servicios de salud, resultando en la co-construcción de los casos bajo condiciones operantes que afectan la permeabilidad y aceptabilidad de los servicios (Dixon-Woods et al., 2006).

En vista de la ausencia de estudios locales que exploren cómo los actores implicados en este proceso enfrentan condiciones que imponen obstáculos para el acceso a servicios, se justifica la necesidad de estudiar las circunstancias que afectan in situ, en los centros de salud primaria, la permeabilidad y aceptabilidad del servicio para las mujeres en el posparto, tomando en cuenta que en este escenario se instala la detección, sospecha y tratamiento de la DPP. Por lo demás, una aproximación situada, etnográfica, a la luz del concepto de 'elegibilidad', permite indagar en determinantes estructurales eminentemente relacionales, como el género.

\section{Método}

Se realizó un estudio cualitativo de tipo etnográfico que hizo factible la observación metódica y estructurada de prácticas sociales para lograr comprender progresivamente 
la rutina diaria de los sujetos estudiados y dotar al acto interpretativo del investigador de un contexto fundamentado en el diálogo y la observación (Taylor y Bogdan, 1994).

El enfoque de género (Rubin, 1986) permitió efectuar una mirada crítica, contextualizada y realista acerca de las problemáticas del acceso a servicios posnatales en el sistema público de salud, contribuyendo al análisis de las determinantes estructurales que condicionan las experiencias de mujeres-madres, usuarias de los centros de salud.

El diseño microetnográfico que se empleó en esta investigación intenciona el carácter exploratorio de un análisis microsocial que facilita la comprensión de las relaciones y vínculos de las personas con su entorno físico y social, desde sus posiciones sociales (Garcez, 1997).

El dispositivo metodológico fue selectivo e intermitente, posibilitando breves, pero continuos, períodos de observación en distintas localidades, analizando intencionadamente aspectos específicos de los procesos sociales para privilegiar la saturación de los datos, distanciándose de una etnografía tradicional (Jeffrey y Troman, 2004).

El trabajo de campo se llevó a cabo en seis establecimientos de APS del Área Metropolitana de Santiago, Chile, durante los meses de enero, febrero y marzo de 2013. Según datos administrativos del MINSAL, los centros seleccionados tenían la mayor cantidad de controles de niño sano en su respectivo servicio de salud.

Se utilizó un muestreo no probabilístico, seleccionando a los sujetos del estudio de manera intencionada, según criterios guiados teóricamente. La muestra se constituyó de beneficiarios/as y proveedores/as que poblaban las salas de espera de los centros seleccionados; los informantes fueron mujeres que asisten a control de niño sano con lactante y profesionales de la salud que realizan la atención.

Los datos fueron recolectados mediante distintos instrumentos: i) observación participante en la sala de espera; ii) entrevistas abiertas de carácter informal realizadas individualmente y en dependencias del centro de salud; y iii) notas de campo, fruto de la observación y de las entrevistas, para aportar información contextual.

La información fue sistematizada en diarios de campo, elaborando una descripción densa (Geertz, 2003) que permitió recabar el máximo de información, construyendo un marco descriptivo en el que, luego de la triangulación de datos, se exponen pautas de comportamiento significativas para los sujetos involucrados. Finalmente, el equipo de investigación realizó un análisis interpretativo del material e identificó tópicos recurrentes.

En términos procedimentales, se obtuvieron los consentimientos informados de los centros de salud y de los participantes. Posteriormente se inició la etapa de inmersión, que consistió en recorridos por las dependencias de los establecimientos y en la observación de pautas de comportamiento de los sujetos estudiados. En segunda instancia la observación se focalizó en aspectos específicos de la producción de cuidados maternos, entrevistando a usuarias (madres) y profesionales para lograr una mayor profundización y complementación de la información recabada. Asimismo, se realizó un acompañamiento, por sala de espera y centro de salud, a ciertas madres. Las notas de campo se tomaron para enriquecer y contextualizar las observaciones, entrevistas y acompañamientos. Después de cada visita, la información se sintetizó en diarios de campo.

\section{Resultados}

Emergieron cuatro categorías principales, que describen las condiciones de producción y reproducción de los cuidados maternos en los centros de salud, identificando algunos elementos que afectan la permeabilidad y aceptabilidad de los servicios, y finalmente, las subjetividades, malestares y otras dinámicas personales de las mujeres en situación posparto que podrían repercutir en la DPP.

\section{El consultorio como espacio de Sociabilidad}

Una mirada a los espacios comunes del establecimiento de salud permite evidenciar cómo la población usuaria se ve envuelta en interacciones que se desarrollan con cierta independencia de la situación de consulta, asistiendo a un lugar que no es un mero lugar de tránsito, ni tampoco se encuentra estrictamente caracterizado por la relación entre prestador/a y beneficiario/a, sino determinado territorialmente y en el cual se reproducen relaciones sociales también regidas por las dinámicas contextuales en que género y poder se entraman de manera desigual y jerarquizada.

Esta situación complejiza la búsqueda de ayuda de las mujeres que asisten con lactantes, ya que las posiciona en una red de intercambio simbólica que no operaría distinto a la cotidianidad habitual, pudiendo ser objeto de juicios de valor al relacionarse con otros actores, y cuyo contenido da cuenta de la mantención de relaciones de género tradicionales.

Asímismo, el diseño del centro de salud juega un papel importante, encausando la sociabilidad y saturando ciertos lugares de mujeres con lactantes - tales como el vacunatorio o la oficina de retiro de alimentos- siendo determinante para la experiencia de estas beneficiarias. 
En los consultorios generales hay zonas exclusivamente destinadas a esto (salud sexual y reproductiva, controles infantiles de rutina o por morbilidad), propiciando encuentros en que lo íntimo compartido es común (como el centralismo del cuidado, de la familia, de sí mismas, o del lactante). Al respecto, una beneficiaria comenta sobre la interacción con otras madres que parecen estar 'en la misma', dando cuenta de cierta complicidad:

“Cuando hablo con otra mamá, me gusta, es como compartir cosas... sirve harto...".

En cambio, en los centros de salud familiar se atiende en un mismo espacio a grupos heterogéneos por distintos motivos de consulta, diversidad que provoca el vuelco de la mirada ajena sobre lo privado, tomando por objeto de intervención las conductas y cuidados del binomio madrehijo/a, evidenciándose una tensión manifiesta en gestos, comentarios, sugerencias e intromisiones. Para la usuaria, esto es fuente de ansiedad, de malestar:

"Las señoras las más viejas como que se sienten con el derecho de decirte que: "el niño tiene chanchitos, que tiene hambre...”. Una se siente presionada...”.

Pareciera ser, de este modo, que la ejecución del cuidado materno, para los otros usuarios, es natural, ya que se lleva a cabo sin esfuerzo y forma parte del sentido común. Como en el testimonio de una mujer respecto de este 'saber ser madre':

"Parece que todos saben [cómo cuidar un bebé]... hasta el guardia... y todos opinan, eso incomoda...”.

\section{El circuito de la mujer-madre}

El itinerario que frecuentan las madres junto a sus lactantes en el centro de salud gira en torno a las instancias en que los cuidados materno-infantiles de salud institucional son provistos:

"Hora con la enfermera por la niña, después voy a retirar la leche, después al vacunatorio... y si me pidieron algún examen, a sacar hora...".

La tensión habitual durante la espera en los espacios comunes se ve acompañada de un semblante apurado y cansado, que se complementa cuando el resultado de la atención es comentado con algún acompañante, generalmente de sexo femenino, haciendo énfasis en cómo la entrega de estos servicios se ensambla con su cotidianidad, refiriendo una lista de asuntos prioritarios en que el cuidado de su salud (de sí misma) no figura:

"Hace falta tiempo, se va todo... pido permiso para venir... y tengo que estar apurada... todo el rato pensando en ello: la niña, la casa, vender [actividad laboral]".

Demostrando signos de desgate:

"Cansa un poco... no, a veces harto... tienes que estar en todas".

También se 'presencia' un grupo de mujeres ausentes, en cuyo reemplazo se presentan, generalmente, figuras femeninas de su círculo familiar -son las otras 'cuidadoras' del lactante, como abuelas o tías de éste-, ausencia que encuentra su fundamento en la ejecución de actividades remuneradas por parte de la madre. En este sentido, la abuela de un lactante dice:

"Mi hija no puede venir [a los controles de niño sano], ella trabaja... o sea, sí, viene, a veces pero tiene que pedir permiso... [ella] me dice que eso es complicado".

Visto como un espacio que acota el tiempo disponible para la ejecución de otras tareas y que coarta los grados de libertad de estas mujeres, el establecimiento es conceptualizado como una carga más dentro de su cotidianidad. Se constata que la conducta al interior del recinto está determinada por los múltiples roles que estas beneficiarias ejecutan, (trabajo, hogar, labores de cuidado), como lo relata otra madre:

"Sólo en las noches me pueden pillar... trabajo en la mañana, en la tarde estudio... y tengo que ver a mis hijos... casi no tengo tiempo para venir...".

\section{Tensiones en la relación entre mujeres y equipos de salud}

Si a la espera en un lugar 'colonizado' por el sentido común que cuestiona las labores de cuidado de las madres, se suma la prisa característica de estas mujeres, entonces, la atención a la salud materno-infantil puede ser terreno fértil para desencuentros entre prestador y beneficiara, sobre 
todo al momento de hacer recomendaciones hacia la diada madre-hijo/a. Al respecto, un enfermero reconoce:

"Me tengo que andar con cuidado... de repente me han llegado quejas: "que las miraste mal, que les hablaste muy alto, que esto y lo otro"... resulta complicado, porque no te das cuenta ni tampoco te lo dicen".

Según los/las profesionales de la salud, la poca o nula adecuación de las consultantes a las normas establecidas por la institución es reflejo de cómo lo mundano ha 'conquistado' el lugar, haciendo hincapié en la parte visible del 'circuito de la mujer-madre', pero desde una mirada aséptica, sintomática e individualizada, que no atisba la cotidianidad subyacente. Como declara una matrona:

“Siempre nerviosas... como apuradas, ansiosas... llegan tarde, corren al mesón... en la consulta se quieren ir rápido".

No obstante, para las usuarias, si la falta de tiempo es un factor importante que impide la asistencia regular, la escasa adecuación de algunas recomendaciones hechas por los/las profesionales impacta negativamente en la motivación para asistir al centro de salud:

“Te dicen consejos... cosas... a veces es dificil hacerlo, casi siempre, como que viene muy de afuera... como que no saben muy bien por lo que una pasa... .".

"Es simpática [hablando de una profesional]... pero tengo tantas cosas que hacer venir acá para que te digan: "mire, usted tiene esto y tiene que hacer esto”... pero si no tengo tiempo”.

\section{Malestar subjetivo}

Transversal a los tópicos ya abordados, emerge un conjunto de testimonios cuyo común denominador es el malestar sentido por las mujeres-madres relacionado a la ejecución de cuidados en condiciones precarias o no equitativas, previamente mencionadas, que exceden su capacidad de respuesta, pudiendo ocasionar una sensación de pérdida de control sobre el cotidiano:

"Tengo que hacer de todo, tantas cosas que no tengo ni tiempo para mis cosas... como que una no sabe para dónde va la cosa ... y los demás, en mi familia... está cada uno ocupado dedicado a sus cuestiones...".

Para algunas, se configura una situación en que la realidad de la función materna adquirió matices imprevistos, carentes de asidero en el discurso común sobre la maternidad. Esta falta de congruencia se resuelve en un malestar que es notorio para terceros, pero censurado, o en la sumisión a la exigencia normativa de ser la madre que otros esperan:

"No todo es como lo pintan... me preguntan por qué no estoy contenta, si debería estarlo... y es que con el quehacer, a veces una se siente chata... "qué lindo el bebé”... y parece que todos saben [cómo cuidar un bebé] (...) asi que mejor, a veces, me quedo calladita no más".

En suma, estas tensiones parecen expresarse en una desazón difusa e indefinible para las puérperas, que en el discurso adquiere el carácter de perplejidad y tedio ante el cúmulo de cargas cotidianas relacionadas a su condición de ser mujer y madre, condición de género, quedando sujeta a la mirada de terceros (intromisiones, juicios, exámenes, intervenciones), delimitando los espacios de las usuarias, subordinándolas.

\section{Discusión y conclusiones}

Este es el primer estudio en nuestro país que explora con una mirada de género desde las relaciones contextuales y psicosociales en que se inserta la atención de las mujeres en posparto, las condiciones que materializan las barreras para la detección de la DPP y sus implicancias para el acceso a tratamiento. Tema que adquiere importancia si pensamos que el sistema de salud público chileno cuenta con tamizaje y acceso universal para esta patología, para lo que se requiere condiciones que permitan garantizar el acceso al uso de servicios y la eficiencia en el uso de recursos.

El enfoque empleado prioriza la observación de las condiciones bajo las que se produce la atención de las madres, y permite rescatar las vivencias de los principales involucrados en el proceso asistencial, prestadores/as y usuarias. Por otra parte, implica una mirada amplia a las relaciones que se tejen en los centros de salud primaria, evidenciando situaciones que exceden a la provisión de servicios, pero que se transforman en determinantes de la atención. 
Los resultados de este estudio sugieren la existencia de barreras para el acceso al servicio, cuando se levanta la sospecha de DPP en las madres. Barreras que se construyen y se presentan en los espacios comunes del centro de salud, como espacio micro-social, y que afectan la aceptabilidad y permeabilidad del servicio, provocando un alto impacto negativo en la relación entre las usuarias y el sistema institucional de cuidados.

En este sentido, el marco referencial que otorga el concepto de 'elegibilidad' es de capital importancia para dotar de poder explicativo a las categorías que emergieron en el análisis, para posicionarlas en el proceso dinámico de búsqueda y entrega de ayuda (Dixon-Woods et al., 2005).

De este modo, la sala de espera del consultorio pasa a ser dominio cotidiano de sus usuarios/as y de sus discursos enraizados en marcos convencionales, sentido común determinado por relaciones de género y poder. Esto tiene implicancias para las mujeres-madres que buscan ayuda, ya que la identificación de su 'malestar' se asentaría en estas representaciones compartidas, que otorgan un marco interpretativo a los signos y síntomas, delimitando aquello que es bueno o malo, sano o enfermo (Young, 1982).

En la misma línea, nuestros resultados coinciden con los de una investigación realizada con madres deprimidas en Gran Bretaña (Edge y MacKian, 2010), que identifica a 'comunidades reflexivas' para dar cuenta cómo la asistencia a los centros de salud está determinada por un pensamiento reflexivo, pero no en el sentido de una entidad individualizada, sino que delimitada socioculturalmente, afectando, inevitablemente, la validación de las manifestaciones patológicas y la búsqueda de ayuda.

Los límites se difuminan, pero lo que parece ponerse en juego en esta 'reflexividad' observada en el consultorio es algo íntimo para las usuarias, su ser madre. Privacidad puesta a prueba por un sentido común que significa el cuidado materno como algo naturalizado, lo que causa ansiedad y disgusto en estas mujeres, que ven cómo las creencias populares sobre la maternidad no se corresponden con su experiencia en este nuevo rol.

La vivencia de malestar y desasosiego que experimentan las mujeres puérperas en el micro-espacio del consultorio también se corresponde con lo sugerido por otros estudios (Beck, 2002), produciéndose acompañada de sentimientos de culpa y desajuste, y alimentando una suerte de círculo vicioso, en que la expresión de la afectividad estaría constreñida por la búsqueda de satisfacción de expectativas. Esto revela el impacto negativo que una construcción idealizada de la maternidad, reforzada socialmente, tiene sobre estas usuarias, cuestión que se corrobora también por resultados de otros estudios (Bilszta, Ericksen, Buist y Milgrom, 2010).

Trazando un paralelo con el proceso de elegibilidad (Dixon-Woods et al., 2006), reconocerse 'apta' para el uso de los servicios de salud implicaría aceptar la existencia de un problema que puede encontrar solución desde el modelo hegemónico de atención en salud, lo que claramente supone enfrentar las creencias naturalizadas de la maternidad y sus restricciones impuestas a la vivencia de comportamientos aparentemente desviados. En consecuencia, este proceso de confirmación de la 'elegibilidad' se extiende más allá de la individualidad de la madre (o de su círculo íntimo), para actualizarse y ser contingente en cada lugar recorrido.

Siguiendo este 'viaje' emprendido por las mujeresmadres puede apreciarse que la asistencia al centro de salud requiere de la movilización de muchos recursos, suponiendo esto un serio impedimento para la continuidad del proceso. El consultorio adquiere una centralidad problemática en el cotidiano de las mujeres, que hace necesaria la coordinación y el uso de redes de apoyo para manejar las distintas responsabilidades o labores que ejecutan estas usuarias: el cuidado de menores de edad, de personas adultas, labores remuneradas, trabajo doméstico, transporte entre estas distintas actividades (dinero y tiempo), etc. Todos estos elementos han sido descritos como barreras para la búsqueda de ayuda, en la literatura internacional (Dennis y Chung-Lee, 2006).

Estas condiciones suponen una múltiple determinación del proceso de 'elegibilidad' en las usuarias, sujeto a la competencia de las distintas labores que llevan a cabo habitualmente. Así, las cargas de la maternidad muchas veces hace que el manejo de la propia salud signifique un costo elevado, o al contrario, un aspecto descuidado, como se constata en algunos testimonios. Esta situación encuentra su correlato en el manejo de la salud en grupos vulnerables, descrito por Dixon-Woods (2005), que tienden a usar los servicios de emergencia ('situaciones de crisis') por sobre los servicios de salud preventiva o promocional, similar a la vivencia de una 'espiral descendiente', en que se ha alcanzado un punto crítico que motiva a las mujeres con DPP a consultar a profesionales de la salud (Beck, 2002).

Llegado a este punto, se podría inferir que el manejo doméstico de la salud de las usuarias como una serie de crisis es sostenido, en parte, por la multiplicidad de cargas de cuidado que recaen sobre las mismas. Llama la atención que este punto no se problematiza en la política sanitaria, parece aceptarse tácitamente que las mujeres-madres ejecuten funciones de cuidadoras domésticas y encargadas de articular los malestares familiares con los servicios asistenciales, y que su disponibilidad no se vea alterada, aún cuando esta 
multiplicidad de roles dé cuenta de su integración a otras áreas (Provoste y Berlagoscky, 2002). Más aún, no se ha explorado cómo conducen su salud, física y mental, bajo estas circunstancias.

Relacionado a lo anterior, se 'observó' un grupo de mujeres que no se presentaban a estos consultorios, madres que se ausentaban a los controles de niño sano debido a sus labores remuneradas y que en lugar de ellas, asistían otras mujeres de su círculo íntimo. Al respecto, surge el cuestionamiento por el grado de integración que existiría entre el trabajo remunerado de estas mujeres y los cuidados de la salud brindados por los consultorios.

En el caso de la DPP, además, habría que preguntarse ¿qué ocurre con la detección de esta patología en aquellas madres que trabajan y que no pueden ser alcanzadas por la asistencia rutinaria de los centros de salud?

De otra parte, no sabemos si el trabajo puede ser o no un factor protector para la DPP. Existe evidencia que sugiere que las madres que no trabajan estarían en un mayor riesgo de padecer DPP (Robertson et al., 2004; Rojas et al., 2010), sin embargo, un reciente informe del Observatorio de Equidad de Género en Salud aborda los efectos negativos que puede tener la 'doble jornada' de trabajo a la que se ven sujetas las mujeres, siendo fuente de tensiones al intentar conciliar sus múltiples tareas (Matamala et al., 2011).

De igual manera, surgen dudas respecto a la figura de las cuidadoras ya que éstas ingresan a los controles de rutina en lugar de la madre, son generalmente familiares de ésta y se hacen cargo de los cuidados del bebé, pasando, probablemente, la mayor parte del día con éste. ¿Qué ocurre con la salud mental de estas mujeres? La calidad de la relación de este binomio debiera ser evaluada, pues puede tener un profundo impacto en el desarrollo del infante. Además, sería necesario tomar en consideración que, según estudios nacionales (Jofré y Alvarado, 2010; Jofré y Mendoza, 2005; Medel, Díaz y Mauro, 2006), las labores de cuidado informal suelen ser llevadas a cabo por mujeres sujetas a distintas situaciones de desventaja (nivel socioeconómico, escolaridad, redes de apoyo), que experimentan una sobrecarga de funciones, lo que conlleva efectos negativos para su salud física y mental. Más aún, tienen conflictos decisionales respecto a su propia salud (Jofré y Mendoza, 2005).

Ante esta evidencia, llama la atención que la investigación en salud mental de cuidadores/as informales de infantes sea un tópico relegado, tomando en cuenta que, en Chile, se ha instalado una reforma de la atención en salud que promueve los cuidados domésticos de los/las enfermos/as (Provoste y Berlagoscky, 2002) y, complementariamente, se ha implementado un sistema de protección integral a la infancia para reducir las inequidades de manera temprana (Silvia y Molina, 2009). Pareciera ser que, en esta planificación, sería necesario recurrir a una mirada de género que pueda problematizar las presunciones naturalizadas acerca de la aparente unidad en la díada madre-hijo.

Continuando con el análisis, el desencuentro entre los/las profesionales de la salud y las usuarias parece ser la consecuencia de un servicio que puede resultar poco permeable y poco aceptable (Dixon-Woods et al., 2005; Magaña et al., 2011).

Así, los testimonios revelan obstáculos debido a condiciones operantes en los consultorios, tales como exigencias normativas ('llegar a la hora') cuyo incumplimiento podría ser visto como inadecuación en la ejecución del rol de 'paciente', sin tomar en cuenta las cargas y demandas cotidianas de las usuarias. Esta incompatibilidad se complementa con las escasas horas asistenciales detectadas en los centros de salud (Chile Crece Contigo-Ministerio de Salud, 2013), que, en su conjunto, pueden dilatar la obtención de ayuda y exigir una mayor movilización de recursos para 'cumplir con las reglas' (Dixon-Woods et al., 2006).

Por otra parte, que las usuarias sientan que las indicaciones de los/las profesionales fueron poco adecuadas, afecta seriamente la continuidad del cuidado en la institución formal de salud. Las beneficiarias suelen poner en la balanza los costos y beneficios de la atención (Finlayson y Downe, 2013), por lo tanto, si han de sortear la carga impuesta por sus múltiples roles y reconocer sus sentimientos de desajuste con el rol materno, para luego experimentar tensiones en su relación con los/las proveedores/as, entonces, como ya se ha mencionado, se reforzará la utilización de los servicios de emergencia, por sobre aquellos dedicados a la salud preventiva.

Que este encuentro se dé en el marco del control de niño sano puede dificultar el abordaje de problemas emocionales relacionados a la ejecución del rol materno, ya que estos controles suelen ser vistos como instancias evaluativas de la salud infantil (un 'producto' de los cuidados maternos), hallazgo concordante con estudios internacionales (ChewGraham, Sharp, Chamberlain, Folkes y Turner, 2009; Slade, Morrell, Rigby, Ricci, Spittlehouse y Brugha, 2010).

Este estudio se vio sujeto a algunas limitaciones. Se emplea un diseño exploratorio, ya que el tópico prácticamente no ha sido abordado en la literatura nacional, por lo que se requiere mayor investigación para poder comparar los resultados a la luz de la evidencia local. Además, se optó por la implementación de un método micro-etnográfico para sortear aquellos aspectos que excedían las posibilidades de la investigación (Garcez, 1997; Jeffrey y Troman, 2004; 
Owen, 1998), tal como contar con un tiempo prolongado de observación.

Por otra parte, se observó la presencia de algunas mujeres inmigrantes que asistían a los controles de niño sano, constatándose un número apreciable de éstas en algunos consultorios. Esta categoría, lamentablemente, no fue tomada en cuenta para no adicionar mayor complejidad en el análisis, empero, reconocemos que este es un tópico que es necesario no relegar y que, según un estudio nacional que evaluó la salud mental y el uso de servicios de salud en inmigrantes (Rojas, Fritsch, Castro, Guajardo, Torres y Díaz, 2011), es una población que experimenta barreras en el acceso a cuidados profesionales en el sistema público de salud.

La presencia de hombres ejerciendo prácticas de cuidado constituyó una minoría, aunque esto se ha visto impulsado por las recientes políticas de protección a la maternidad y paternidad, por lo que podemos pensar, entonces, que queda un largo camino por recorrer en cuanto a la equidad de género.

Finalmente, para futuras líneas de investigación se sugiere indagar en torno a los procesos de salud, enfermedad y atención posteriores al parto desde una perspectiva de género exhaustiva, que contemple el impacto que el nacimiento de un hijo tiene en la salud mental de hombres y mujeres.

\section{Referencias}

Alvarado, R., Rojas, M., Monardes, J., Perucca, E., Neves, E., ... Olea, E. (2000). Cuadros depresivos en el posparto en una cohorte de embarazadas: construcción de un modelo causal. Revista chilena de neuropsiquiatría, 38, 84-93.

Araya, R., Rojas, G., Fritsch, R., Acuña, J. y Lewis, G. (2001). Common mental disorders in Santiago, Chile: prevalence and socio-demographic correlates. The British Journal of Psychiatry, 178, 228-33.

Beck, C. T. (2002). Postpartum Depression: A Metasynthesis. Qual Health Res, 12, 453-472.

Bilszta, J., Ericksen, J., Buist, A. y Milgrom, J. (2010). Women's experience of postnatal depression - beliefs and attitudes as barriers to care. Australian Journal of Advanced Nursing, 27, 44-54.

Busfield, J. (1988). Mental illness as social product or social construct: a contradiction in feminists' arguments? Sociology of Health \& Illness, $10,521-542$.

Chew-Graham, C., Sharp, D., Chamberlain, E., Folkes, L. y Turner, K. (2009). Disclosure of symptoms of postnatal depression, the perspectives of health professionals and women: a qualitative study. $B M C$ Family Practice, 10, 7.

Chile Crece Contigo-Ministerio de Salud (2013). Informe sobre análisis de la situación de uso de servicios y acceso a tratamiento de la depresión posparto en centros de atención primaria en salud de la Región Metropolitana. Santiago: Unidad Chile Crece Contigo-MINSAL.

Dennis, C. L. y Chung-Lee, L. (2006). Postpartum depression help-seeking barriers and maternal treatment preferences: a qualitative systematic review. Birth, 33, 323-31.

Dixon-Woods, M., Cavers, D., Agarwal, S., Annandale, E., Arthur, A., ... Harvey, J. (2006). Conducting a critical interpretive synthesis of the literature on access to healthcare by vulnerable groups. BMC Medical Research Methodology, 6, 35.

Dixon-Woods, M., Kirk, D., Agarwal, S., Annandale, E., Arthur, T., ... Harvey, J. (2005). Vulnerable groups and access to health care: a critical interpretive review. Recuperado desde http:/www.netscc.ac.uk/hsdr/ files/project/SDO_FR_08-1210-025_V01.pdf.

Drago, M. (2006). La reforma al sistema de salud chileno desde la perspectiva de los derechos humanos. CEPAL - SERIE Politicas sociales, 121, 1-94.

Edge, D. y MacKian, S. C. (2010). Ethnicity and mental health encounters in primary care: help-seeking and help-giving for perinatal depression among Black Caribbean women in the UK. Ethnicity \& Health, 15, 93-111.

Finlayson, K. y Downe, S. (2013). Why do women not use antenatal services in low- and middle-income countries? A meta-synthesis of qualitative studies. PLoS Medicine, 10, e10D1373. doi: 10.1371/ journal.pmed.1001373.

Garcez, P. (1997). Microethnography. Encyclopedia of Language and Education, 8, 187-196.

Geertz, C. (2003). La interpretación de las culturas. Barcelona: Gedisa S.A. Jadresic, E. y Araya, R. (1995). Prevalencia de depresión posparto y factores asociados en Santiago, Chile. Revista Médica de Chile, 123, 649-99.

Jeffrey, B. y Troman, G. (2004). Time for ethnography. British Educational Research Journal, 30, 535-548.

Jofré, V. y Alvarado, O. (2010). Evaluación de la sobrecarga de cuidadores/as informales. Ciencia y Enfermería, 16, 111-120.

Jofré, V. y Mendoza, S. (2005). Toma de decisiones en salud en mujeres cuidadores informales. Ciencia y Enfermería, 11, 37-49.

Magaña, I., Calquín, C., Silva, S. y García, M. (2011). Diversidad Familiar, Relaciones de Género y Producción de Cuidados en Salud en el Modelo de Salud Familiar: Análisis de Caso en un CESFAM de la Región Metropolitana, Chile. Terapia Psicológica, 29, 33-42.

Matamala, M. I., Eguiguren, P. y Díaz, X. (2011). Tensiones y silencios en la reforma de la salud 2011: género y derechos ausentes. Santiago: OPS.

Medel, J., Díaz, X. y Mauro, A. (2006). Cuidadoras de la vida: visibilización de los costos de la producción de salud en el hogar. Impacto sobre el trabajo total de las mujeres. Centros de Estudios de la Mujer-CEM.

Ministerio de Salud (s.f). Programa Nacional de Diagnóstico y Tratamiento de la Depresión. Recuperado desde http://www.minsal.gob.cl/ $\mathrm{portal} / \mathrm{url} / \mathrm{page} / \mathrm{minsalcl} / \mathrm{g}$ _proteccion/g_salud_mental/programanacdiagytratadepre.html.

Ministerio de Salud (2006). Segundo Régimen de Garantías Explícitas en Salud. Santiago: MINSAL.

Ministerio de Salud (2013). Guía Clínica de la Depresión en personas de 15 años y más. Santiago: MINSAL.

Owen, B. (1998). In the Mix: Struggle and Survival in a Women's Prison. New York: SUNY Press.

Patel, V., Araya, R., de Lima, M., Ludermir, A. y Todd, C. (1999). Women, poverty and common mental disorders in four restructuring societies. Social Science \& Medicine, 49, 1461-71.

Póo, A. M., Espejo, C., Godoy, C., Gualda, M., Hernández, T. y Pérez, C. (2008). Prevalencia y factores de riesgo asociados a la depresión posparto en puérperas de un Centro de Atención Primaria en el Sur de Chile. Revista Médica de Chile, 136, 44-52.

Provoste, P. y Berlagoscky, F. (2002). Género, Equidad y Reforma de la Salud en Chile. Modelo de atención y género: Las condiciones socioculturales de la Reforma de la Salud en Chile. OPS/OMS Serie Género y Reforma de Salud, 4, 1-23.

Risco, L., Jadresic, E., Galleguillos, T., Garay, J. 1., González, M. y Hasbún, J. (2002). Depresión postparto: alta frecuencia en puérperas Chilenas, detección precoz, seguimiento y factores de riesgo. Psiquiatría y Salud Integral, 2, 61-6.

Robertson, E., Grace, S., Wallington, T. y Stewart, D.E. (2004). Antenatal risk factors for postpartum depression: a synthesis of recent literature. General Hospital Psychiatry, 26, 289-95.

Rojas, G., Fritsch, R., Solís, J., González, M., Guajardo, V. y Araya, R. (2006). Calidad de vida de mujeres deprimidas en el posparto. Revista Médica de Chile, 134, 713-20. 
Rojas, G., Fritsch, R., Solís, J., Jadresic, E., Castillo, C., ... González, M. (2007). Treatment of postnatal depression in low-income mothers in primary-care clinics in Santiago, Chile: a randomised controlled trial. The Lancet, 10, 1629-37.

Rojas, G., Fritsch, R., Guajardo, V., Rojas, F., Barroilhet, S. y Jadresic, E. (2010). Caracterización de madres deprimidas en el posparto. Revista Médica de Chile, 138, 536-42.

Rojas, G., Fritsch, R., Castro, A., Guajardo, V., Torres, P. y Díaz, B. (2011). Trastornos mentales comunes y uso de servicios de salud en población inmigrante. Revista Médica de Chile, 139, 1298-1304.

Rubin, G. (1986). El tráfico de mujeres: notas sobre la "economía política" del sexo. Nueva Antropología, 8, 95-145.

Silvia, V. y Molina, H. (2009). Cuatro Años Creciendo Juntos: Memoria de la instalación del sistema de Protección Integral de la Infancia, Chile Crece Contigo. Santiago: Minsal.

Slade, P., Morrell, C. J., Rigby, A., Ricci, K., Spittlehouse, J. y Brugha, T. (2010). Postnatal women's experiences of management of depressive symptoms: a qualitative study. The British Journal of General Practice, 60, e440-8. doi: 10.3399/bjgp10X532611.

Taylor, S. J. y Bogdan, R. (1994). Introducción a los métodos cualitativos de investigación. La búsqueda de significados. Barcelona: Ediciones Paidós Ibérica.

Wachs, T. D., Black, M. M. y Engle, P. L. (2009). Maternal depression: a global threat to children's health, development, and behavior and to human rights. Child Development Perspectives, 3, 51-59.

Young, A. (1982). The anthropologies of illness and sickness. The Annual Review of Anthropology, 11, 257-85. 
